\title{
SEMICLASSICAL ANALYSIS OF TWO- AND THREE-SPIN ANTIFERROMAGNETS AND ANYONS ON A SPHERE
}

\author{
DIPTIMAN SEN* \\ Centre for Theoretical Studies, Indian Institute of Science, \\ Bangalore 560012, India
}

\begin{abstract}
We do a semiclassical analysis for two or three spins which are coupled antiferromagnetically to each other. The semiclassical wave functions transform correctly under permutations of the spins if one takes into account the Wess-Zumino term present in the path integral for spins. The Wess-Zumino term here is a total derivative which has no effect on the energy spectrum. The semiclassical problem is related to that of anyons moving on a sphere with the statistics parameter $\theta$ being $2 \pi S$ for two spins and $3 \pi S$ for three spins. Finally, we present a novel way of deriving the semiclassical wave functions from the spin wave functions.
\end{abstract}

PACS numbers: 75.10.Jm, 03.65.Sq, 74.20.Kk

* E-mail: diptiman@cts.iisc.ernet.in 
This Letter illustrates three different ideas using a simple quantum mechanical model. These ideas, which have been studied recently in other contexts, include a semiclassical treatment of quantum spins for large values of the spin $S$ [ $1-5$ ], a Wess-Zumino (WZ) term which is present in the path integral for spins [2,6], and the possibility of fractional statistics on the sphere $[7,8]$. We consider two or three spins coupled antiferromagnetically. Although a naive semiclassical analysis reproduces the low-lying energies and degeneracies correctly, there is a marked difference between the ways in which the naive semiclassical and spin wave functions transform under the exchange of any two spins. The complete symmetry group is the permutation group $S_{N}$ of $N$ spins in the quantum theory, and the braid group $B_{N}$ on the sphere in the naive semiclassical theory. We relate this difference in transformation properties to a WZ term which appears in the coherent state path integral Lagrangian for spins. This term treats each spin as a charged particle moving on a sphere which has a magnetic monopole of strength $4 \pi S$ at its center. Once this term is taken into account, the modified semiclassical wave functions have the correct transformation properties under spin permutations. We can think about the semiclassical problem in terms of $N$ anyons moving on a sphere with the statistics parameter $\theta$ (which is defined modulo $2 \pi$ ) being $2 \pi S$ for $N=2$ and $3 \pi S$ for $N=3$. Thus the naive semiclassical wave functions for three half-integer spins exhibit semionic statistics. At the end, we will verify our analysis by directly constructing the correct semiclassical wave functions from the spin wave functions .

Consider first an antiferromagnetic Hamiltonian for two spins $H=\left(\vec{S}_{1}+\right.$ $\left.\vec{S}_{2}\right)^{2} \equiv \vec{l}^{2}$. For any value of the spin $S$, the energies are $l(l+1)$ with a degeneracy $2 l+1$ where $l=0,1,2, \ldots, 2 S$. The three- $j$ symbols have the symmetry [9] 


$$
\left(\begin{array}{ccc}
S & S & l \\
m_{1} & m_{2} & m
\end{array}\right)=(-1)^{2 S+l}\left(\begin{array}{ccc}
S & S & l \\
m_{2} & m_{1} & m
\end{array}\right)
$$

where $m_{1}, m_{2}$ and $m$ denote eigenvalues of $\left(\vec{S}_{1}\right)_{3},\left(\vec{S}_{2}\right)_{3}$ and $l_{3}$ respectively. Hence, under the exchange $P_{12} \equiv \vec{S}_{1} \leftrightarrow \vec{S}_{2}$, the wave functions transform by the phase $\left(P_{12}\right)_{q u}=(-1)^{2 S+l}$ where the subscript 'qu' denotes quantum .

Now consider a semiclassical treatment of this problem. For $S>>1$, we introduce a vector $\vec{\phi}=\left(\vec{S}_{1}-\vec{S}_{2}\right) / 2 S$. This satisfies

$$
\begin{aligned}
\vec{l} \cdot \vec{\phi} & =0 \\
\text { and } \vec{\phi}^{2} & =1+\frac{1}{S}-\frac{\vec{l}^{2}}{4 S^{2}}
\end{aligned}
$$

For low-lying excitations (i.e. $l<<S$ ), we see from (1) that $\vec{\phi}$ is an unit vector. The naive semiclassical Lagrangian is

$$
\mathcal{L}=\frac{1}{4} \dot{\vec{\phi}}^{2}
$$

with the constraint $\vec{\phi}^{2}=1$. Canonical quantization of (2) reproduces the above Hamiltonian except that $-\vec{l}^{2}$ is now given by the Laplacian $\vec{\nabla}^{2}$ on a sphere. The semiclassical energies are therefore $l(l+1)$ with degeneracy $2 l+1$, and the naive wave functions are the spherical harmonics $Y_{l, m}(\alpha, \beta)$. (Here $(\alpha, \beta)$ are the polar angles such that $\vec{\phi}=(\sin \alpha \cos \beta, \sin \alpha \sin \beta, \cos \alpha)$. Namely, the direction of spin (or particle) 1 has the coordinates $(\alpha, \beta)$ while particle 2 is at the point $(\pi-\alpha, \pi+\beta)$ where $0 \leq \alpha \leq \pi, 0 \leq \beta<2 \pi)$. Semiclassically, $l$ can be any non-negative integer. This spectrum agrees with the exact one for $0 \leq l \leq 2 S$. It is interesting that the semiclassical energies and degeneracies are correct even if $S$ is not much greater than one and $l$ is not much less than $S$. Under the exchange of the two spins, $\vec{\phi} \rightarrow-\vec{\phi}$. The wave functions $Y_{l, m}$ then transform by the phase $\left(P_{12}\right)_{n s c}=(-1)^{l}$ where the subscript 'nsc' denotes naive semiclassical . 
The difference of $(-1)^{2 S}$ between $\left(P_{12}\right)_{q u}$ and $\left(P_{12}\right)_{n s c}$ can be explained as follows. The two-spin problem can be semiclassically thought of as two particles moving on a sphere with the Hamiltonian forcing them to lie at antipodal points for low energies. Under an exchange, the two particles will together trace out a closed curve which encloses a solid angle $2 \pi$. Now, it is known that the path integral for spins contains a WZ term which makes each particle see a magnetic monopole of strength $4 \pi S$ at the center of the sphere [2,6 ]. A particle which goes around a closed curve enclosing a solid angle $\Omega$ picks up an Aharonov-Bohm phase exp $(i \Omega S)$. Thus an exchange of the two particles produces a phase $\exp (i 2 \pi S)$.

To be explicit, the WZ term equals $S \dot{\beta}(1+\cos \alpha)$ for particle 1 if we choose the Dirac string of the monopole to pass through the north pole $\alpha=0$. The WZ term for particle 2 is then $S \dot{\beta}(1-\cos \alpha)$. The sum of the two is

$$
\mathcal{L}_{W Z}=2 S \dot{\beta}
$$

which is a total derivative. The correct semiclassical wave functions are therefore $\exp (i 2 \beta S) Y_{l, m}(\alpha, \beta)$. Note that since the phase factor $\eta=\exp (i 2 \beta S)$ is singlevalued (except at the points $\alpha=0$ or $\pi$ when one of the particles lies on the Dirac string), the energy spectrum is unaffected by (3). But the new semiclassical wave functions do show the correct exchange phase $\left(P_{12}\right)_{q u}$ due to the factor $\eta$.

To summarize the semiclassical picture, the particles behave like anyons on a sphere with the parameter $\theta=2 \pi S$. For $N$ anyons on a sphere, $\theta$ is only allowed to have the $2(N-1)$ values given by $\pi p /(N-1)$, where $p=0,1,2, \ldots, 2 N-3[8]$. So we may hope to find a truly anyonic behavior (i.e. $\theta \neq 0$ or $\pi$ ) if $N=3$. We therefore turn to the more interesting problem of three spins.

The Hamiltonian $H=\left(\vec{S}_{1}+\vec{S}_{2}+\vec{S}_{3}\right)^{2} \equiv \vec{l}^{2}$ can be shown to have the low-lying spectrum $l(l+1)$ with degeneracy $(2 l+1)^{2}$ for $0 \leq l \leq S$. (For $l>S$, the 
expression for degeneracy is different). Here $l$ takes integer or half-integer values if $S$ is an integer or half-integer respectively. For any $l$, let us consider the $2 l+1$ states which have the eigenvalue $l_{3}=l$, and study their transformation properties under the permutation group $S_{3}$ of the three spins. (Since the group operations of $S_{3}$ commute with the total spin operators $\vec{l}$, the same transformation properties will hold for other values of $l_{3}$ also). We have only examined small values of $S$ and $l$, namely, $0 \leq l \leq S \leq 2$. For integer $S$, we find that these $2 l+1$ states fall into $l+1$ irreducible representations (IR) of $S_{3}$ consisting of $l$ doublets and one singlet. Under the exchange of any two spins, the singlet picks up the phase $(P)_{q u}=(-1)^{S+l}$. (For example, the ground state $(l=0)$ has a totally symmetric wave function if $S$ is an even integer, and an antisymmetric wave function if $S$ is an odd integer). For half-integer $S$, the $2 l+1$ states with $l_{3}=l$ fall into $l+1 / 2$ doublets. Under any exchange, the doublets always transform by a $2 \times 2$ matrix whose eigenvalues are \pm 1 .

Some of the above statements can be understood using the two-spin results. To obtain a total spin $l$ with three spins, the spin of two of them, say $\vec{S}_{1}$ and $\vec{S}_{2}$, must add up to values lying in the range $S-l, \ldots, S+l$. (This explains the $(2 l+1)$-fold degeneracy for a given value of $l_{3}$ ). Under the subgroup $P_{12}$, the exchange phases are therefore $(-1)^{3 S-l}, \ldots,(-1)^{3 S+l}$. It then follows that for half-integer $S$, there are $l+1 / 2$ states with $P_{12}=-1$ and $l+1 / 2$ with $P_{12}=1$, while for integer $S$, there are $l$ states with $P_{12}=(-1)^{S+l+1}$ and $l+1$ with $P_{12}=(-1)^{S+l}$. Of course, it needs more work to derive the singlet and doublet structure under the full group $S_{3}$.

Now we do a semiclassical analysis. For large $S$, we introduce two vectors [ 4 ] 


$$
\begin{aligned}
\vec{\phi}_{1} & =\frac{\vec{S}_{1}-\vec{S}_{2}}{\sqrt{3} S} \\
\text { and } \quad \vec{\phi}_{2} & =\frac{\vec{S}_{1}+\vec{S}_{2}-2 \vec{S}_{3}}{3 S}
\end{aligned}
$$

One can again derive identities similar to (1) which show that for $S$ much greater than both $l$ and $1, \vec{\phi}_{1}^{2}=\vec{\phi}_{2}^{2}=1$ and $\vec{\phi}_{1} \cdot \vec{\phi}_{2}=0$. We introduce a third vector $\vec{\phi}_{3}=\vec{\phi}_{1} \times \vec{\phi}_{2}$ and define an $S O(3)$ matrix $\underline{R}$ whose columns are given by the three vectors $\vec{\phi}_{1}, \vec{\phi}_{2}$ and $\vec{\phi}_{3}$ respectively. The total spin $\vec{l}$ transforms $\underline{R}$ from the left. Thus $\left[l_{i}, \underline{R}\right]=-T_{i} \underline{R}$ where $i=1,2,3$ and the matrices $T_{i}$ have the elements $\left(T_{i}\right)_{j k}=-i \epsilon_{i j k}$.

There is a two-to-one mapping from the manifold of $S U(2)$, namely the hypersphere $S^{3}$, to the matrices $\underline{R}$. (This is made explicit in Eq. (13) below). We parametrize points on $S^{3}$ by an $S U(2)$ matrix $\underline{V}=x_{4}+i \vec{\sigma} \cdot \vec{x}$ where $\vec{\sigma}$ are the Pauli matrices and $x_{4}^{2}+x_{1}^{2}+x_{2}^{2}+x_{3}^{2}=1$. For later use, we define

$$
\begin{aligned}
z_{1} & =x_{1}+i x_{2}=\sin a e^{i b} \\
\text { and } z_{2} & =x_{3}+i x_{4}=\cos a e^{i c}
\end{aligned}
$$

where $0 \leq a \leq \pi / 2$ and $0 \leq b, c<2 \pi$. The symmetry group of the manifold $S^{3}$ is $S O(4)=S U(2) \times S U(2) / Z_{2}$. These two $S U(2)$ 's are generated by matrices $\vec{l}$ and $\vec{l}^{\prime}$ which act on $\underline{R}$ (or $\underline{V}$ ) from the left and right respectively [ 10 ]. (The two can be thought of as rotations about a set of axes which is either space-fixed or body-fixed). On $\underline{R}$, a rotation acts as an orthogonal matrix $\underline{O}=\exp (i \epsilon \hat{n} \cdot \vec{T})$ while on $\underline{V}$, the corresponding action is by an unitary matrix $\underline{U}=\exp (i \epsilon \hat{n} \cdot \vec{\sigma} / 2)$ where $\hat{n}$ is an unit vector and $\epsilon$ is the rotation angle. (Notice that we are using the same symbol $\vec{l}$ to denote both the quantum spin operators and the semiclassical angular momenta which act from the left. It will be clear from the context which one we mean). 
The semiclassical Lagrangian can now be shown to be $[4,11]$

$$
\mathcal{L}=\frac{1}{8} \operatorname{tr} \underline{\dot{R}}^{T} \underline{\dot{R}}=\frac{1}{2} \operatorname{tr} \underline{\dot{V}}^{\dagger} \underline{\dot{V}}
$$

where $\underline{R}^{T}=\underline{R}^{-1}$ and $\underline{V}^{\dagger}=\underline{V}^{-1}$. Canonical quantization of this yields $H=-\vec{\nabla}^{2} / 4$ where $\vec{\nabla}^{2}$ is the Laplacian on $S^{3}$. The normalization in Eq. (6) is fixed by considering small fluctuations near the identity. $\underline{R}=I_{3}+i 2 \vec{\epsilon} \cdot \vec{T}$ corresponds to $\underline{V}=I_{2}+i \vec{\epsilon} \cdot \vec{\sigma}$ which is near the north pole of $S^{3}$, namely, $x_{4}=1$. (Here $I_{n}$ denotes the $n \times n$ identity matrix). Then (6) becomes $\mathcal{L}=\dot{\vec{\epsilon}}^{2}$, the Hamiltonian is $\vec{\Pi}_{\epsilon}^{2} / 4$, and the Laplacian on $S^{3}$ is $\vec{\nabla}_{\epsilon}^{2}=-\vec{\Pi}_{\epsilon}^{2}$.

On $S^{3}$, the left operators $\vec{l}$ have the form [ 10 ]

$$
\begin{aligned}
& l_{3}=\frac{1}{2}\left(z_{1} \partial_{1}+z_{2} \partial_{2}-z_{1}^{\star} \partial_{1}^{\star}-z_{2}^{\star} \partial_{2}^{\star}\right)=\frac{1}{2 i}\left(\frac{\partial}{\partial b}+\frac{\partial}{\partial c}\right) \\
& l_{+}=z_{2} \partial_{1}^{\star}-z_{1} \partial_{2}^{\star} \text { and } l_{-}=z_{1}^{\star} \partial_{2}-z_{2}^{\star} \partial_{1}
\end{aligned}
$$

while the right operators $\vec{l}^{\prime}$ are

$$
\begin{aligned}
& l_{3}{ }^{\prime}=\frac{1}{2}\left(z_{1} \partial_{1}-z_{2} \partial_{2}-z_{1}^{\star} \partial_{1}^{\star}+z_{2}^{\star} \partial_{2}^{\star}\right)=\frac{1}{2 i}\left(\frac{\partial}{\partial b}-\frac{\partial}{\partial c}\right) \\
& l_{+}{ }^{\prime}=z_{2}^{\star} \partial_{1}^{\star}-z_{1} \partial_{2} \text { and } l_{-}{ }^{\prime}=z_{1}^{\star} \partial_{2}^{\star}-z_{2} \partial_{1}
\end{aligned}
$$

where $\partial_{i}$ denotes $\partial / \partial z_{i}$. The Laplacian is $\vec{\nabla}^{2}=-4 \vec{l}^{2}=-4 \vec{l}^{\prime 2}$. The harmonic functions on $S^{3}$ transform as the representation ( $\left.l, l\right)$ under $S U(2) \times S U(2)$. Here $l$ can be an integer or half-integer. The degeneracy is $(2 l+1)^{2}$. (For instance, the first five harmonics are 1 for $l=0$, and $z_{1}, z_{2}, z_{1}^{\star}$ and $z_{2}^{\star}$ for $\left.l=1 / 2\right)$. The Laplacian acting on these harmonics gives $-4 l(l+1)$. Under a $2 \pi$ rotation of the spins about any axis, $\underline{R} \rightarrow \underline{R}, \quad \underline{V} \rightarrow-\underline{V}$ and the harmonics transform by $(-1)^{2 l}$. So we have to choose $l$ to be an integer (or half-integer) if $S$ is an integer (or half-integer). Thus the semiclassical theory reproduces the correct spectrum for $0 \leq l \leq S$. The proper manifold for semiclassical quantization is therefore $S O(3)$ for integer $S$ and $S U(2)$ for half-integer $S$ as we might have expected for an odd number of spins. 
The $2 l+1$ semiclassical wave functions with $l_{3}=l$ are $z_{1}^{2 l}, z_{1}^{2 l-1} z_{2}, \ldots, z_{2}^{2 l}$. An exchange of any two spins is equivalent to a $180^{\circ}$ rotation about the third spin which is a matrix acting on $\underline{R}$ or $\underline{V}$ from the right. (For $\underline{V}$, one has to further specify whether the rotation is clockwise or anticlockwise as the corresponding matrices differ by a minus sign). Under any one of the three possible $180^{\circ}$ rotations, we find that

$$
\left(\begin{array}{l}
z_{1} \\
z_{2}
\end{array}\right) \rightarrow \underline{P}\left(\begin{array}{l}
z_{1} \\
z_{2}
\end{array}\right)
$$

where $\underline{P}$ is an off-diagonal unitary matrix whose eigenvalues are $\pm i$, not \pm 1 . For example, an exchange of $\vec{S}_{1}$ and $\vec{S}_{2}$ corresponds to multiplying $\underline{R}$ from the right by

$$
\left(\begin{array}{ccc}
-1 & 0 & 0 \\
0 & 1 & 0 \\
0 & 0 & -1
\end{array}\right)
$$

and $\underline{V}$ from the right by $i \sigma^{2}$ or $-i \sigma^{2}$. So the matrix in (8) is $\underline{P}_{12}=i \sigma_{2}$ or $-i \sigma_{2}$.

Hence, for half-integer $l$, the $2 l+1$ functions given above fall into $l+1 / 2$ doublets each transforming irreducibly under the braid group $B_{3}$, namely,

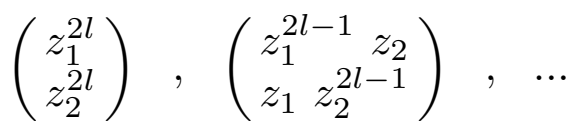

Under any exchange, these doublets transform with eigenvalues $\pm i$. For integer $l$, on the other hand, the $2 l+1$ states fall into $l$ doublets as in Eq. (9), but there is also a singlet given by $z_{1}^{l} z_{2}^{l}$. Under any exchange, the doublets transform with eigenvalues \pm 1 while the singlet picks up the phase $(P)_{n s c}=(-1)^{l}$. Thus in all cases, the eigenvalues of the (one- or two-dimensional) exchange matrices $\left(\underline{P}_{i j}\right)_{n s c}$ differ from those of $\left(\underline{P}_{i j}\right)_{q u}$ by the phase $\exp (i 3 \pi S)$.

We can understand this by using the WZ term and a typical exchange shown in Fig. 1. The antiferromagnetic Hamiltonian forces the three particles to lie $120^{\circ}$ apart 
on a sphere. Assume that $\vec{S}_{3}$ is fixed along the south pole $(0,0,-1)$ and exchange $\vec{S}_{1}$ and $\vec{S}_{2}$ by a $180^{\circ}$ rotation. The resultant closed curve on the sphere encloses the solid angle $\Omega=2 \pi\left(1+\cos 60^{\circ}\right)=3 \pi$. Hence the Aharonov-Bohm phase picked up is $\exp (i 3 \pi S)$. Note that the sense of the exchange is important if $S$ is an half-integer. In this Letter, however, we will not be precise about whether the argument of the phase is $3 \pi S$ or $-3 \pi S$.

The symmetry group of three particles placed $120^{\circ}$ apart on the sphere is the braid group $B_{3}$. A one- or two-dimensional IR of $B_{3}$ can be obtained by taking an IR of the permutation group $S_{3}$ and making it anyonic by multiplying the appropriate matrices by $\exp (i \theta)$ for a clockwise exchange of any two particles. What we have shown above is that the anyon parameter $\theta$ is equal to $\pm 3 \pi S$.

We now study how these naive wave functions get modified if the WZ term is included in the Lagrangian. We first parametrize the three classical spins $\vec{S}_{n}$ in terms of the orthonormal vectors $\vec{\phi}_{n}$ given by

$$
\begin{aligned}
\vec{\phi}_{3} & =(\sin \alpha \cos \beta, \sin \alpha \sin \beta, \cos \alpha) \\
\vec{\phi}_{1} & =(-\cos \alpha \cos \beta,-\cos \alpha \sin \beta, \sin \alpha) \\
\text { and } \quad \vec{\phi}_{2} & =(\sin \beta,-\cos \beta, 0)
\end{aligned}
$$

Then

$$
\vec{S}_{n} / S=\sin \left(\gamma+\frac{2 \pi n}{3}\right) \vec{\phi}_{1}-\cos \left(\gamma+\frac{2 \pi n}{3}\right) \vec{\phi}_{2}
$$

for $n=1,2,3$. Now we find that the WZ term obtained by adding up the contributions from the three spins is again a total derivative. Indeed,

$$
\mathcal{L}_{W Z}=S \frac{d}{d t}\left[3 \beta-3 \gamma-i \ln \left(\frac{\tan ^{3}(\alpha / 2)-i \exp (i 3 \gamma)}{\tan ^{3}(\alpha / 2)+i \exp (-i 3 \gamma)}\right)\right]
$$

Next, we rewrite the matrix $\underline{R}$ in terms of the $S^{3}$ coordinates (5). This takes the form

$$
\underline{R}=\left(\begin{array}{ccc}
1-2\left(x_{2}^{2}+x_{3}^{2}\right) & 2\left(x_{1} x_{2}+x_{3} x_{4}\right) & 2\left(x_{3} x_{1}-x_{2} x_{4}\right) \\
2\left(x_{1} x_{2}-x_{3} x_{4}\right) & 1-2\left(x_{3}^{2}+x_{1}^{2}\right) & 2\left(x_{2} x_{3}+x_{1} x_{4}\right) \\
2\left(x_{3} x_{1}+x_{2} x_{4}\right) & 2\left(x_{2} x_{3}-x_{1} x_{4}\right) & 1-2\left(x_{1}^{2}+x_{2}^{2}\right)
\end{array}\right)
$$


A comparison then shows that $\alpha=2 a, \quad \beta=b+c$ and $\gamma=c-b$. The WZ term is thus

$$
\mathcal{L}_{W Z}=-i S \frac{d}{d t} \ln \left(\frac{z_{1}^{3}-i z_{2}^{3}}{z_{1}^{\star 3}+i z_{2}^{\star 3}}\right)
$$

Hence the correct semiclassical wave functions are obtained by multiplying the naive wave functions by the phase factor

$$
\eta=\left(\frac{z_{1}^{3}-i z_{2}^{3}}{z_{1}^{\star 3}+i z_{2}^{\star 3}}\right)^{S}
$$

(Our choice for the direction of the Dirac string and the expression for $\eta$ seem to break rotational invariance. However, one can define new angular momenta by the unitary transformation $\vec{l} \rightarrow \eta \vec{l} \eta^{-1}$ ).

We see that $\eta$ is single-valued on $S^{3}$ (unless $z_{1}^{3}=i z_{2}^{3}$ which corresponds to one of the particles being at the north pole of the sphere). Hence the spectrum remains unchanged. But the phase factor restores the correct permutation properties to the wave functions. For instance, under the exchange $P_{12}, z_{1} \rightarrow z_{2}$ and $z_{2} \rightarrow-z_{1}$. Then $\eta$ changes by $\exp (-i 3 \pi S)$ as required. (See the discussion following Eq. (21) below. If $P_{12}$ is taken to transform $z_{1} \rightarrow-z_{2}$ and $z_{2} \rightarrow z_{1}$, then $\eta$ changes by $\exp (i 3 \pi S))$.

Finally, we turn to a derivation of the semiclassical wave functions from the spin wave functions for $S=1 / 2$. For the direction $\hat{n}=(\sin \alpha \cos \beta, \sin \alpha \sin \beta, \cos \alpha)$, the eigenvector of $\hat{n} \cdot \vec{\sigma} / 2$ with eigenvalue $1 / 2$ is given by

$$
|\alpha, \beta\rangle=\left(\begin{array}{c}
\cos (\alpha / 2) \exp (-i \beta) \\
\sin (\alpha / 2)
\end{array}\right)
$$

The phase is chosen such that (16) is ill-defined only at the north pole. The ket $|\vec{\phi}\rangle$ for the two-spin problem is defined to be the tensor product

$$
|\vec{\phi}\rangle \equiv|\alpha, \beta\rangle \otimes|\pi-\alpha, \pi+\beta\rangle
$$


where the first and second factors denote the vectors (16) for spins 1 and 2 respectively. The exact ground state with $l=l_{3}=0$ has the wave function

$$
\left|\psi_{0,0}\right\rangle=\frac{1}{\sqrt{2}}\left(\begin{array}{l}
1 \\
0
\end{array}\right) \otimes\left(\begin{array}{l}
0 \\
1
\end{array}\right)-\frac{1}{\sqrt{2}}\left(\begin{array}{l}
0 \\
1
\end{array}\right) \otimes\left(\begin{array}{l}
1 \\
0
\end{array}\right)
$$

We then define the semiclassical wave function to be the amplitude $\left\langle\vec{\phi} \mid \psi_{0,0}\right\rangle$ which turns out to be $\exp (i \beta) Y_{0,0}(\alpha, \beta) / \sqrt{2}$. Similarly, the three excited states with $l=1$ have the wave functions $\left\langle\vec{\phi} \mid \psi_{1, m}\right\rangle=\exp (i \beta) Y_{1, m} / \sqrt{6}$. (The $Y_{l, m}$ are normalised according to the measure $d \vec{\phi}=\sin \alpha d \alpha d \beta / 4 \pi)$.

For the three-spin problem, we define the ket $|\underline{V}\rangle$ as the tensor product

$$
|\underline{V}\rangle=\left|\alpha_{1}, \beta_{1}\right\rangle \otimes\left|\alpha_{2}, \beta_{2}\right\rangle \otimes\left|\alpha_{3}, \beta_{3}\right\rangle
$$

where the three kets $\left|\alpha_{n}, \beta_{n}\right\rangle$ can be deduced from Eq. (11). To be explicit, the bra $\left\langle\alpha_{n}, \beta_{n}\right|$ takes the form

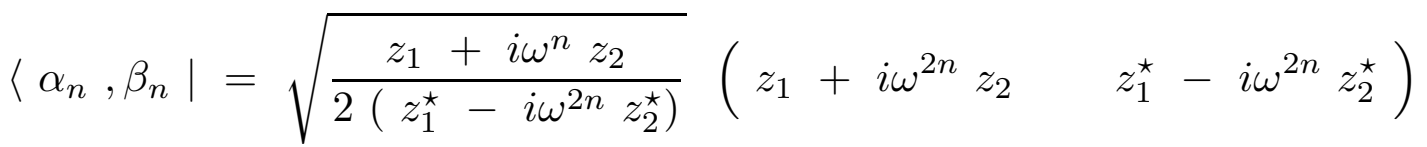

where $\omega=\exp (i 2 \pi / 3)$. It is then clear that any 3 -spin wave function $|\psi\rangle$ will have an amplitude $\langle\underline{V} \mid \psi\rangle$ which is a polynomial in $z_{i}$ and $z_{i}^{\star}$ multiplied by the phase $\eta$ in Eq. (15). For instance, consider the two ground states with $l=l_{3}=1 / 2$. Their wave functions

$$
\begin{aligned}
\left|\psi_{1}\right\rangle= & \frac{1}{\sqrt{2}}\left(\begin{array}{l}
1 \\
0
\end{array}\right) \otimes\left(\begin{array}{l}
0 \\
1
\end{array}\right) \otimes\left(\begin{array}{l}
1 \\
0
\end{array}\right)-\frac{1}{\sqrt{2}}\left(\begin{array}{l}
0 \\
1
\end{array}\right) \otimes\left(\begin{array}{l}
1 \\
0
\end{array}\right) \otimes\left(\begin{array}{l}
1 \\
0
\end{array}\right) \text { and } \\
\left|\psi_{2}\right\rangle= & \frac{1}{\sqrt{6}}\left(\begin{array}{l}
1 \\
0
\end{array}\right) \otimes\left(\begin{array}{l}
0 \\
1
\end{array}\right) \otimes\left(\begin{array}{l}
1 \\
0
\end{array}\right)+\frac{1}{\sqrt{6}}\left(\begin{array}{l}
0 \\
1
\end{array}\right) \otimes\left(\begin{array}{l}
1 \\
0
\end{array}\right) \otimes\left(\begin{array}{l}
1 \\
0
\end{array}\right) \\
& -\frac{2}{\sqrt{6}}\left(\begin{array}{l}
1 \\
0
\end{array}\right) \otimes\left(\begin{array}{l}
1 \\
0
\end{array}\right) \otimes\left(\begin{array}{l}
0 \\
1
\end{array}\right)
\end{aligned}
$$

transform under the exchange $P_{12}$ with phases -1 and 1 respectively. One then finds that

$$
\begin{aligned}
\left\langle\underline{V} \mid \psi_{1}\right\rangle & =\frac{\sqrt{3}}{4}\left(z_{2}+i z_{1}\right) \eta \\
\text { and }\left\langle\underline{V} \mid \psi_{2}\right\rangle & =\frac{\sqrt{3}}{4}\left(z_{1}+i z_{2}\right) \eta
\end{aligned}
$$


If $P_{12}$ takes $z_{1} \rightarrow z_{2}$ and $z_{2} \rightarrow-z_{1}$, then $\eta \rightarrow i \eta$ (that is, $\eta \rightarrow \eta \exp (-i 3 \pi S))$. Hence $\left\langle\underline{V} \mid \psi_{1}\right\rangle$ and $\left\langle\underline{V} \mid \psi_{2}\right\rangle$ transform correctly under $P_{12}$.

This construction can be generalized to any spin $S$. Let us work in a basis in which $\left(\vec{S}_{n}\right)_{3}$ is diagonal. The key ingredient is the eigenvector $|\alpha, \beta\rangle$ (with eigenvalue $S$ ) of the matrix $\hat{n} \cdot \vec{S}$. The $m^{\text {th }}$ entry of the column $|\alpha, \beta\rangle$ is given by $[9]$

$$
|\alpha, \beta\rangle_{m}=\sqrt{\left(\begin{array}{c}
2 S \\
S+m
\end{array}\right)}\left(\cos \frac{\alpha}{2}\right)^{S+m}\left(\sin \frac{\alpha}{2}\right)^{S-m} e^{-i(S+m) \beta}
$$

where $m$ takes the values $S, S-1, \ldots,-S$ from top to bottom. This is welldefined everywhere except at the north pole. Then the two-spin ket in (17) produces wave functions of the form

$$
\left\langle\vec{\phi} \mid \psi_{l, m}\right\rangle=N_{S, l, m} e^{i 2 S \beta} Y_{l, m}
$$

where $N_{S, l, m}$ is a normalization constant. To prove (23), we observe that in terms of the Euler angles $(\alpha, \beta, \gamma)$, the vectors $\exp (i S \beta)|\alpha, \beta\rangle$ and $\exp (i S \beta)|\pi-\alpha, \pi+\beta\rangle$ are given by the first and last columns of the rotation matrix $\mathcal{D}^{(S)}(\alpha, \beta, \gamma)$ respectively. These two columns are proportional to $\exp (-i S \gamma)$ and $\exp (i S \gamma)$. The $\gamma$-dependence cancels when we take a direct product of the two. Thus $\exp (i 2 S \beta)|\vec{\phi}\rangle$ is given by a $(2 S+1)^{2}$-dimensional column of the direct product $\mathcal{D}^{(S)}(\alpha, \beta, 0) \otimes$ $\mathcal{D}^{(S)}(\alpha, \beta, 0)$. The transformation properties of this object under rotations and consequently the differential equation satisfied by it are then precisely the same as those of the spherical harmonics $Y_{l, m}(\alpha, \beta)$. (If there had been a dependence on $\gamma$, then the transformations and the differential equation would have been different since they would involve $\partial / \partial \gamma[9])$. Thus,

$$
e^{-i 2 S \beta}\left\langle\vec{\phi}|\vec{l}| \psi_{l, m}\right\rangle=\vec{l} e^{-i 2 S \beta}\left\langle\vec{\phi} \mid \psi_{l, m}\right\rangle
$$


On the left hand side of (24), $\vec{l}=\vec{S}_{1}+\vec{S}_{2}$ is a matrix which acts on $\left|\psi_{l, m}\right\rangle$ while on the right hand side, $\vec{l}$ is a differential operator acting on $\exp (-i 2 S \beta)\left\langle\vec{\phi} \mid \psi_{l, m}\right\rangle$. (For example, $l_{3}=-i \partial / \partial \beta$ ). Then Eq. (23) follows from the fact that $\left|\psi_{l, m}\right\rangle$ has $\vec{l}^{2}=l(l+1)$ and $l_{3}=m$.

The constants $N_{S, l, m} \equiv N_{S, l}$ in (23) are independent of $m$ due to rotational symmetry. We can choose $N_{S, l}$ to be real and positive. The completeness of the $\left|\psi_{l, m}\right\rangle$ and the fact that $\langle\vec{\phi} \mid \vec{\phi}\rangle=1$ means that

$$
\sum_{l=0}^{2 S}(2 l+1) N_{S, l}^{2}=1
$$

Note that the $(2 S+1)^{2} \times(2 S+1)^{2}$ matrix

$$
M=\int d \vec{\phi}|\vec{\phi}\rangle\langle\vec{\phi}|
$$

is not proportional to the identity. The orthonormality of the $\left|\psi_{l, m}\right\rangle$ implies that the eigenvalues of $M$ are given by the constants $N_{S, l}^{2}$. An explicit formula for $N_{S, l}$ can be derived by considering the state with $l_{3}=l$. The corresponding three- $j$ symbols are given by

$\left(\begin{array}{ccc}S & S & l \\ m & l-m & -l\end{array}\right)=(-1)^{S+l-m}\left[\left(\begin{array}{c}2 l \\ l\end{array}\right) \frac{(2 S-l) !(S+m) !(S+l-m) !}{(2 S+l+1) !(S-m) !(S-l+m) !}\right]^{1 / 2}$

Using this one finds that

$$
e^{-i 2 S \beta}\left\langle\vec{\phi} \mid \psi_{l, l}\right\rangle \sim Y_{l, l}(\alpha, \beta)
$$

and the constant of proportionality determines

$$
N_{S, l}^{2}=\frac{(2 S) !(2 S) !}{(2 S+l+1) !(2 S-l) !}
$$

For the three-spin problem, the ket in Eq. (18) produces the wave functions 
$\left\langle\underline{V} \mid \psi_{l, m}\right\rangle$ which are given by polynomials in $z_{i}$ and $z_{i}^{\star}$ times the phase in (15). From (16), (19) and (22), it is clear that the polynomial is even for integer $S$ and odd for half-integer $S$. An analysis similar to the one for the two-spin case will show that the polynomials are now given by harmonic functions on $S^{3}$. We will however omit the details here.

It is worth noting that the semiclassical analysis in this Letter holds for values of spin as small as $1 / 2$. It would be interesting to extend these considerations to more than three spins [ 5 ] or even to a spin chain. We often know, by either numerical or exact methods, the way in which the quantum ground state and low-lying excitations of antiferromagnetic spin chains transform under discrete symmetries like parity (defined as the reflection of the chain about one site). One might ask how this is related to the symmetry properties of the semiclassical field theories which are typically some non-linear sigma models.

\section{Acknowledgments}

I thank Sumathi Rao for numerous discussions. 


\section{References}

1. F. D. M. Haldane, Phys. Rev. Lett. 50, 1153 (1983) and Phys. Lett. 93 A, 464 (1983).

2. E. Fradkin, Field Theories of Condensed Matter Systems (Addison-Wesley, New York, 1991).

3. I. Affleck, in Fields, Strings and Critical Phenomena, Les Houches, 1988, ed. E. Brezin and J. Zinn-Justin (North Holland, Amsterdam, 1990).

4. T. Dombre and N. Read, Phys. Rev. B 39, 6797 (1989); S. Rao and D. Sen, IISc preprint IISc-CTS-92-9 (unpublished).

5. D. Loss, D. P. DiVincenzo and G. Grinstein, Phys. Rev. Lett. 69, 3232 (1992); J. von Delft and C. L. Henley, Phys. Rev. Lett. 69, 3236 (1992).

6. F. D. M. Haldane, Phys. Rev. Lett. 61, 1029 (1988) and Phys. Rev. Lett. 57, 1488 (1986); T. Dombre and N. Read, Phys. Rev. B 38, 7181 (1988); E. Fradkin and M. Stone, Phys. Rev. B 38, 7215 (1988).

7. F. Wilczek, Fractional Statistics and Anyon Superconductivity (World Scientific, Singapore, 1990).

8. T. D. Imbo and J. March-Russell, Phys. Lett. 252 B, 84 (1990); T. Einarsson, Phys. Rev. Lett. 64, 1995 (1990).

9. E. P. Wigner, Group Theory (Academic Press, New York, 1959); J. D. Talman, Special Functions (W. A. Benjamin, New York, 1968).

10. D. Sen, J. Math. Phys. 27, 472 (1986); R. E. Cutkosky, J. Math. Phys. 25, 939 (1984).

11. A. P. Balachandran, G. Marmo, B.-S. Skagerstam and A. Stern, Classical Topology and Quantum States (World Scientific, Singapore, 1991). 


\section{Figure Caption}

1. Three particles placed $120^{\circ}$ apart on a sphere. Exchanging particles 1 and 2 keeping particle 3 fixed traces out a closed curve which encloses the shaded area. The complement of this area covers the solid angle $3 \pi$. 